\title{
Practice Spotlight: Volunteer Pharmacists in Sub-Saharan Africa
}

\author{
Kyle J Wilby, BSP, PharmD, ACPR \\ Doctor of Pharmacy Student (at time of writing) \\ Faculty of Pharmaceutical Sciences \\ The University of British Columbia \\ Vancouver, British Columbia \\ Freddy E Kitutu, BSc(Pharm), MPS \\ Assistant Lecturer \\ School of Pharmacy, College of Health Sciences \\ Makerere University \\ Kampala, Uganda \\ Samuel Boakye \\ Placement Coordinator \\ BaseCamp International Centers \\ Accra, Ghana
}

$A \begin{aligned} & \text { rmed with knowledge, textbooks, medical supplies, and } \\ & \text { mosquito repellant, Canadian pharmacists depart each year }\end{aligned}$ for international volunteer experiences in different areas of the world. Sub-Saharan Africa is a common destination for health care professionals, because of the high prevalence of disease and shortages of health care workers. ${ }^{1}$ In recent years, pharmacists and student pharmacists have been working with local African organizations to optimize the distribution and provision of pharmacy-related services in this region. These volunteers bring particular knowledge, skills, and attitudes that can positively contribute to humanitarian efforts. Although many different types and durations of volunteer projects are available, this article focuses on pharmacists who take on medium- to longer-term assignments (minimum 4-week placements) in clinic and hospital settings.

The need for optimizing pharmacy-related services in developing countries has increased with worldwide improvements in access to medications. ${ }^{2}$ Major challenges facing these countries include health worker shortages, limited opportunities for training and continuing education, prevalence of counterfeit medications, traditional noncollaborative practice settings, and limited access to drug information resources and literature. As with any organization encountering challenges, external consultation and assistance can be of great value. Volunteer pharmacists and student pharmacists from countries such as Canada can offer knowledge and experience to assist local organizations as they attempt to improve services, advance practice, and maximize positive patient outcomes.

Practice sites for volunteers in sub-Saharan Africa range from large urban teaching hospitals to small rural clinics. One example of a large urban institution is Mulago Hospital in Kampala, Uganda, a 1500-bed public tertiary care hospital that serves as a teaching hospital for the medical and pharmacy schools at Makerere University. It is a hub of academic, research, and service collaborations with universities and health institutions in other African countries, as well as Canada, the United States, and the United Kingdom. Its distinctive environment affords volunteer pharmacists and student pharmacists the opportunity for a rich interactive learning experience.

The hospital is currently served by 7 full-time pharmacists and up to 60 pharmacy interns. These pharmacists are responsible for dispensing services, as well as ward rounds and clinical services in their respective areas. They also play critical roles in general pharmaceutical management activities focused on ensuring availability of medicines, providing patient-focused services, and meeting the drug information needs of other health care providers. Every day, these highly knowledgeable and motivated pharmacists face many challenges, including high patient loads, staff shortages, limited access to reference materials, and inconsistent medication supplies.

Volunteer pharmacists can have major sustainable impacts in this setting by initiating interventions (such as clinical training of pharmacy interns), facilitating case-based therapeutic discussions with pharmacy staff, developing and co-presenting drug information rounds to medical teams, and attending ward rounds with pharmacists to help prioritize and focus drugrelated problems. By working directly with members of the hospital's pharmacy team and focusing on education and training, volunteers can ensure that their interventions become sustainable.

At the other end of the spectrum is Nsutaman Polyclinic, a small rural health centre located in the town of Nsuta in the Ashanti Region of Ghana. This publicly funded clinic serves the 
population of Nsuta and people in a number of smaller villages in the same district. Basic medical and maternity services are offered at the clinic, which houses a small dispensary staffed by a pharmacy technician and nursing assistants. Drug supplies are limited, and storage of vaccines and sensitive drug products is difficult because of the harsh climate and frequent shortages of electricity and water. Medications are counted and dispensed in small plastic bags with minimal dosing instructions. The pharmacy staff is mostly responsible for dispensing medications, compounding, and ensuring appropriate storage conditions.

At this clinic, volunteer pharmacists work with pharmacy staff to create drug information resource binders, develop counselling services for essential drug information, assist with inventory management, give presentations to clinic staff on health promotion and medication safety, and work with the maternity staff to create reference material for the use of medications in pregnancy and lactation. Volunteers are also involved with outreach visits to rural villages, where they assist with the administration and documentation of vaccinations.

Mulago Hospital and Nsutaman Polyclinic are only 2 examples of practice sites for volunteer pharmacists in subSaharan Africa. However, the types of volunteer activities discussed here are applicable to many settings in the region. In addition, beneficial effects may be realized even after the volunteers depart, through continued collaboration in research and training, awareness campaigns, training and recruitment of future volunteers, and the creation of knowledge-sharing networks between health care workers in the host and originator countries.

Opportunities for volunteer pharmacists in sub-Saharan Africa will continue to increase as access to medication expands and shortages of health care workers persist. Additionally, student pharmacists are starting to travel to Ghana, Uganda, and other countries for clinical clerkships and research rotations. Before choosing an organization through which to volunteer, prospective pharmacist volunteers are advised to thoroughly research options in terms of credibility, funding, past volunteer reviews, experience working with medical professionals, and method of allocating the program fee (if any). Direct contact and interaction with staff at the placement site before departure are also recommended, to begin needs assessments and to establish the volunteer's role within the organization. By taking these steps, volunteers increase their chances of having positive experiences that are beneficial for all parties involved.

Volunteering in sub-Saharan Africa can be a very rewarding experience and may lead to long-lasting friendships, exchanges, and collaborations. International travel and information technology have made it easier for pharmacists around the globe to come together in achieving their common goals of advancing pharmacy practice and improving patient care. The knowledge and experiences gained from working with practitioners in sub-Saharan Africa are invaluable and can help to improve pharmacy practice in Canada. It should always be remembered that health care workers based in sub-Saharan Africa have just as much (if not more) to give to volunteers as the volunteers have to offer in return.

\section{References}

1. Laleman G, Kegels G, Marchal B, Van der Roost D, Bogaert I, Van Damme W. The contribution of international health volunteers to the health workforce in sub-Saharan Africa. Hum Resour Health 2007;5:19. Available from: www.human-resources-health.com/content/5/1/19

2. Anderson C, Bates I, Beck D, Penick Brock T, Futter B, Mercer H, et al. The WHO UNESCO FIP pharmacy education taskforce. Hum Resour Health 2009;7:45. Available from: www.human-resources-health.com/ content/7/1/45

About the authors: All of the authors have extensive experience developing, implementing, and evaluating pharmacist and student pharmacist volunteer placements in sub-Saharan Africa.

The Practice Spotlight series highlights the accomplishments of Canadian pharmacists with unique practices in hospitals and related health care settings. If you have a unique or innovative practice, or you know someone else who should be profiled, please submit your contact information to Mary Ensom, Editor of CJHP (cjhpedit@cshp.ca), and one of our Associate Editors will be in touch with you. 